\title{
MOLECULAR EPIDEMIOLOGY OF MULTIDRUG RESISTANT ACINETOBACTER BAUMANNII CLINICAL ISOLATES FROM TWO BULGARIAN HOSPITALS
}

\author{
Temenuga Stoeva $^{1}$, Paul Higgins ${ }^{2}$, Kalina Bojkova ${ }^{1}$, Harald Seifert $^{2}$ \\ ${ }^{1}$ Department of Microbiology, Medical University of Varna \\ ${ }^{2}$ Institute for Medical Microbiology, Immunology and Hygiene, University of Cologne
}

\begin{abstract}
OBJECTIVES: To investigate the molecular epidemiology of multidrug resistant (MDR) Acinetobacter baumannii isolates collected from patients in two Bulgarian Hospitals (H1, H2) during the period 20052012.

MATERIALS AND METHODS: A total of 93 nonduplicate MDR A. baumannii isolates collected from Intensive care units (ICUs) and surgical wards were studied. Antimicrobial susceptibility was tested by VITEK 2 (bioMérieux, Marcy l'Etoile) and Phoenix (BD) and the results were interpreted according to the current CLSI guidelines. Detection of carbapenemase encoding genes was performed by PCR. Isolates were genotyped by PFGE and rep-PCR DiversiLab system (bioMerieux) and compared against a library of $A$. baumannii strains representing the international clones (IC) 1-8.

RESULTS: Twenty seven isolates were carbapenem resistant and were associated with OXA-23 and OXA-58 enzymes. The majority of the investigated isolates $(n=65)$ clustered with ICs 1,2 and 8 . Twenty five isolates from $\mathrm{H} 2$, collected during the period 2005-2007, did not cluster with any of the representative strains for the ICs 1-8 and formed a separate unique clone, which was found to persist in 2012.

CONCLUSIONS: Our results reveal the epidemic and endemic potential of $A$. baumannii as well as persistence of clones over time. Multidrug resistance in A. baumannii from the investigated Bulgarian hospitals was associated with intrahospital dissemination of IC1, 2 and 8 and with unique local clones.
\end{abstract}

Keywords: Acinetobacter baumannii, multidrug resistance, epidemiology, international clones

Address for correspondence:

Assoc. Prof. Temenuga Zhekova Stoeva, MD PhD

Department of Microbiology

Medical University of Varna

55 Marin Drinov Str.

Tel: +35952978 538

e-mail: temenuga.stoeva@abv.bg

Received: May 14, 2014

Accepted: September 10, 2014

\section{INTRODUCTION}

Acinetobacter baumannii is a major nosocomial pathogen, particularly in the intensive care unit (ICU) setting. This bacterial species is characterized by its resistance to multiple antimicrobial agents, propensity for clonal spread and involvement in numerous hospital outbreaks. Eight A. baumannii international clones (IC 1-8) have been identified. IC 2 is the most widespread and is often associated with carbapenem resistance (1). 
A. baumannii is one of the most commonly isolated multidrug resistant (MDR) pathogens in the University Hospital "Saint Marina" (H1) and Municipal Hospital "Saint Anna" (H2) in Varna, Bulgaria during the last decade. The objective of this study was to investigate the molecular epidemiology of MDR A. baumannii isolates collected from patients in the two hospitals during the period 2005/2007 and 2010/2012 with the aim to identify the predominant clonal lineages circulating in the hospitals and their mechanism of carbapenem resistance.

\section{MATERIAL AND METHODS}

A total of 93 nonduplicate MDR isolates of A. baumannii, obtained from patients admitted in ICUs (58\%) and surgical wards (42\%), were investigated. The isolates were recovered predominantly from wound specimens (46\%) and tracheal aspirates $(42 \%)$. Sixty four isolates were collected in the period 2005-2007; 3 and 26 isolates - in 2010 and 2012 respectively. Species identification was performed by gyrB multiplex PCR (2). Antimicrobial susceptibility was tested by VITEK 2 (bioMérieux, Marcy l'Etoile, France) and Phoenix (BD) and the results were interpreted according to the current CLSI guidelines (3). The isolates were genotyped by PFGE and repPCR DiversiLab Microbial Typing System (bioMérieux, Nürtingen, Germany) and compared against a library of A. baumannii strains representing the International clones 1-8 $(4,5)$. Detection of carbapenemase encoding genes $\left(b l a_{\text {OXA-23-like }}, b l a_{\text {OXA-24-like, }}, b l a-\right.$ OXA-58-like, $\left.b l a_{\text {OXA-51-like, }}, b l a_{\mathrm{IMP}}, b l a_{\mathrm{VIM}}, b l a_{\mathrm{NDM}}\right)$ was performed by PCR $(6,8)$.

\section{RESULTS AND DISCUSSION}

Twenty six isolates (28\%) were imipenem and meropenem resistant and resistant to most of the antibiotics tested, except colistin. One isolate (blaOXA-58-like positive) demonstrated resistance to imipenem $(\mathrm{MIC}=16 \mu \mathrm{g} / \mathrm{ml})$, but susceptibility to meropenem $(\mathrm{MIC}=4 \mu \mathrm{g} / \mathrm{ml})$. Most isolates were resistant to $\beta$-lactams other than carbapenems, including piperacillin - $100 \%$; ceftazidime - $98.9 \%$; cefepime - $97.8 \%$ and ampicillin/sulbactam - $92.5 \%$, as well as to non- $\beta$-lactam antibiotics: gentamicin $-91.4 \%$; amikacin $-87 \%$; ciprofloxacin $-91.4 \%$ and trimethoprim/sulfamethoxazole $-87 \%$.
In the group of isolates from $\mathrm{H} 2(\mathrm{n}=68), \mathrm{PFGE}$ of the ApaI-digested genomic DNA identified 6 pulsotypes (A-F), two of which were predominant $(A, B)$ (Table 1). Rep-PCR confirmed this clustering. Pulsotype A was represented by 25 carbapenem susceptible (CS) isolates, recovered from five different wards during the period 2005-2007: ICU, Orthopedic surgery, Thoracic surgery, II General surgery and Neurosurgery. Representative isolates for pulsotype A did not cluster with any of the major international clonal lineages and formed a separate, unique clone, dominant during the period 2005-2007. This clone was found to persist in 2012, but was represented by carbapenem resistant (CR) isolate (Fig. 1).

Pulsotype B was represented by $22 \mathrm{CS}$ and one CR isolate, all collected during 2005-2007 from Orthopedic surgery, I and II General surgery wards and the ICU. Two environmental isolates (working surfaces and sheets) from the ICU and Orthopedic surgery were found to express identical PFGE pulsotype $\mathrm{B}$ as that of the clinical isolates, demonstrating the role of the hospital environment as an important factor for transmission and dissemination of this nosocomial pathogen. Representative isolates for pulsotype B were found to correspond to IC1 (Figure 1). In 2012 no isolates were found to cluster with IC1. Both dominant clones A and B have spread in parallel among several wards of the hospital during the studied period, indicating nosocomial cross transmission.

Pulsotype D was detected in 9 CS isolates, all recovered from five different wards (ICU, Gynecology, Orthopedic surgery, I and II General surgery wards) but only in 2005. Representative isolates for this pulsotype cluster with IC8 (Figure 1). Isolates belonging to IC8 were not found in 2012.

Pulsotype E was identified in 5 CS isolates, collected from ICU, Orthopedic surgery and II General Surgery ward during the period 2006-2007. Representative isolates of pulsotype E cluster with IC2. Although the first IC2 isolate was recovered in 2006 in Orthopedic surgery, this clone was also detected in 2012 but was already represented by CR A. baumannii isolates. The isolates from H1 (22 CR and 3 $\mathrm{CS})$, recovered predominantly from the surgical ICU $(n=23)$ during the period 2010-2012, also clustered 
Table 1. Pulsotype, year of isolation and clustering with the major ICs.

\begin{tabular}{|c|c|c|c|c|c|c|c|}
\hline \multicolumn{2}{|c|}{ Pulsotype } & n & IC & $\begin{array}{l}\text { Year(s) of } \\
\text { isolation }\end{array}$ & Wards & $\begin{array}{c}\text { n } \\
\text { CR }\end{array}$ & $\begin{array}{c}\text { Acquired } \\
\boldsymbol{b l a}_{\mathrm{OXA}}\end{array}$ \\
\hline H1 & - & 25 & IC2 & $2010-2012$ & ICU & 22 & OXA - 23 \\
\hline $\mathrm{H} 2$ & A & 26 & unc & $\begin{array}{c}2005-2007 \\
2012\end{array}$ & $\begin{array}{l}\text { ICU, OS, TS } \\
\text { GSII, NS }\end{array}$ & 1 & OXA - 23 \\
\hline & B & 23 & IC1 & $2005-2007$ & $\begin{array}{c}\text { ICU, OS, GSI, } \\
\text { GSII }\end{array}$ & 1 & OXA - 58 \\
\hline & $\mathrm{C}$ & 1 & unc & 2005 & OS & - & \\
\hline & D & 9 & IC8 & 2005 & $\begin{array}{c}\text { ICU, G, OS, } \\
\text { GSI, GSII }\end{array}$ & - & \\
\hline & $\mathrm{E}$ & 8 & IC2 & $\begin{array}{c}2006-2007 \\
2012\end{array}$ & ICU, OS, GSII & 3 & OXA - 23 \\
\hline & F & 1 & Unc & 2005 & GSI & - & \\
\hline
\end{tabular}

H1, Hospital 1; H2, Hospital 2; CR, carbapenem resistant; Unc, unclustered with IC; ICU, intensive care unit; TS, Thoracic surgery, GS I, I General surgery; GSII, II General surgery; NS, Neurosurgery; OS, Orthopedic surgery; G, Gynecology.

with IC2 (Figure 1). Pulsotype $\mathrm{C}$ and F from $\mathrm{H} 2$ represented single isolates.

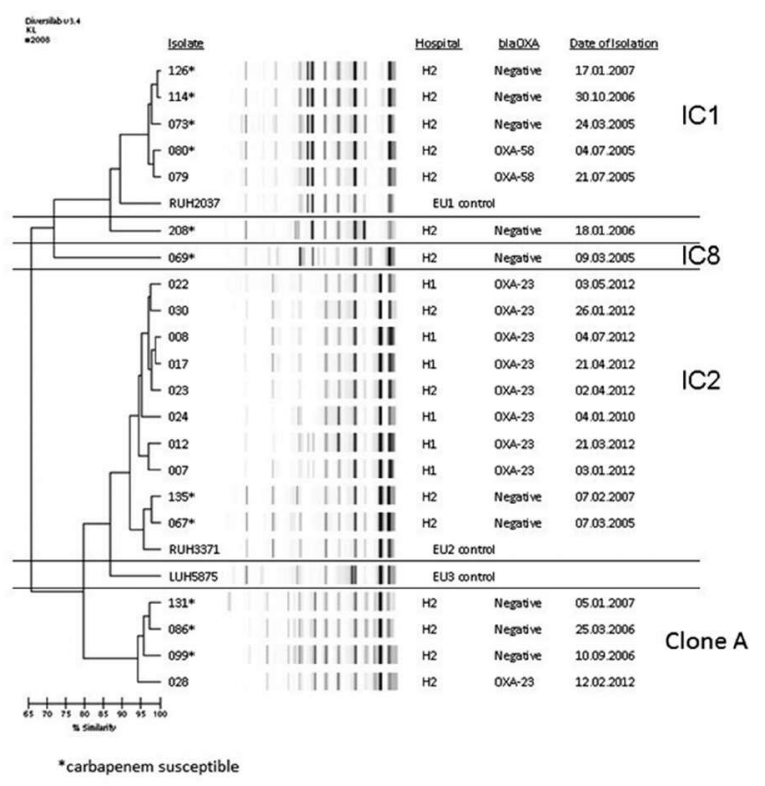

Fig. 1. Dendrogram and rep-PCR profiles of $21 \mathrm{~A}$. baumannii isolates from $\mathrm{H} 1$ and $\mathrm{H} 2$ and clustering with the major ICs.

All isolates had the intrinsic $b l a_{\text {OXA-51-like }}$ gene. All CR A. baumannii were found to harbour an acquired $b l a_{\text {OXA }}$ gene; $b l a_{\text {OXA-23-like }}$ was detected in twenty six CR isolates and $b l a_{\text {OXA-58-like }}$ in one CR isolate. $b l a_{\text {OXA-58-like }}$ was also detected in one CS isolate. In 2005/2007, the only acquired $b l a_{\text {OXA-like }}$ in $A$. baumannii was $b l a_{\text {OXA-58- }}$ like . In 2012, only isolates harbouring bla $a_{\text {OXA-23-like }}$ were detected. With one exception, no acquired $b l a_{\text {OXA-like }}$ was detected in CS isolates.

\section{CONCLUSIONS}

This study demonstrates the epidemic and endemic potential of A. baumannii. A total of $70 \%$ of the investigated isolates from both hospitals cluster with IC1, 2 and 8, confirming the wide geographical distribution of these epidemic clonal lineages. In 2005-2007, MDR in A. baumannii in H2 was associated with coexistence and intrahospital dissemination of the unique local clone A and with IC1, IC2 and IC8. Clone A and IC2 were found still to persist in 2012. In this regard, the problem of MDR A. baumannii is not only related to the international clonal lineages but also to other successfully disseminated genotypes. In this study, carbapenem resistance in the investigated Bulgarian hospitals, mediated mainly by the acquired OXA- 23 carbapenemase, was associated with both persisting hospital clones $\mathrm{A}$ and IC2.

\section{Acknowledgements}

We thank M. Grigorova for providing the isolates and supporting this study. 


\section{REFERENCES}

1. Higgins PG, Dammhayn C, Hackel M, Seifert

H. Global spread of carbapenem - resistant

Acinetobacter baumannii. J Antimicrob

Chemother 2010; 65: 233 - 238.

2. Higgins $\mathrm{PG}$, Wisplinghoff $\mathrm{H}, \mathrm{Krut} \mathrm{O}$, Seifert $\mathrm{H}$.

A PCR-based method to differentiate between Acinetobacter baumannii and Acinetobacter genomic species 13TU. Clin Microbiol Infect 2007; 13:1199-1201.

3. Clinical and Laboratory Standards Institute. Methods for dilution antimicrobial susceptibility tests for bacteria that grow aerobically, 7 th edn. Approved Standard M7-A7. Wayne, PA: CLSI, 2007.

4. 4. Healy M, Huong J, Bittner T, Lising M, Frye $S$, Raza $S$ et al. Microbial DNA typing by automated repetitive-sequenced -based PCR. J Clin Microbiol 2005; 43: 199-207.

5. Seifert H, Dolzani L, Bressan R, van der Reijden, van Strijen B, Stefanik D et al. Standartization and interlaboratory reproducibility assessment of pulsed-field gel electrophoresis-generated fingerprints of Acinetobacter baumannii. J Clin Microbiol 2005; 43: 4328-4335.

6. Pfeifer $Y$, Wilharm G, Zander E, Wichelhaus T, Gottig S, Hunfeld KP et al. Molecular characterization of blaNDM-1 in an Acinetobacter baumannii strain isolated in Germany in 2007. J Antimicrob Chemother 2011; 66 (9): 1998-2001.

7. Senda K, Arakawa Y, Ichiyama S, Nakashima K, Ito $\mathrm{H}$, Ohsuka $\mathrm{S}$ et al. PCR detection of metallo$\beta$-lactamase gene (blaIMP) in gram-negative rods resistant to broad-spectrum $\beta$ - lactams. J Clin Microbiol 1996; 34: 2909-2913.

8. Woodford N, Ellington MJ, Coelho JM, Turton JF, Ward ME, Brown S et al. Multiplex PCR for genes encoding prevalent OXA carbapenemases in Acinetobacter spp. Int J Antimicrob Agents 2006; 27: 351-353. 